\title{
Treatment with TRPV2 antibody ameliorates the severity of heart failure in dilated cardiomyopathic hamsters
}

\author{
Yuko Iwata, Madoka Hirayama, Shin Ito, Masafumi Kitakaze \\ National Cerebral and Cardiovascular Center, Japan
}

Dilated cardiomyopathy (DCM) is characterized by both ventricular dilation and contractile dysfunction, and the pathophysiology of DCM is mainly considered as progressive myocardial damages attributable to the abnormal myocardial $\mathrm{Ca}^{2+}$ handling. Transient receptor potential cation channel, subfamily V, member 2 (TRPV2) has been previously suggested as a principal candidate for $\mathrm{Ca}^{2+}$ entry pathways, which seem to be a potential therapeutic target for DCM. However, there are no known TRPV2-specific inhibitors to date.

Here, we produced antibodies against TRPV2, which interact with the TRPV2 extracellular site and inhibit the $\mathrm{Ca}^{2+}$ influx via TRPV2. Since these antibodies had no effects on the other members of TRP family such as TRPV1 and TRPC1, we tested the therapeutic efficacy of TRPV2 inhibition in the muscular dystrophy/cardiomyopathy hamsters (J2N-k) and found that abnormal $\mathrm{Ca}^{2+}$ influx and stretch-induced muscle damage in J2N-k myocytes were significantly reduced by these antibodies. Furthermore, we observed that the treatment with one of the antibodies by intraperitoneal injections at a dose of $0.5 \mathrm{mg} / \mathrm{kg}$ twice a week for 2 weeks, ameliorated the severity of cardiac dysfunction (Fractional Shortening (\%); $37.0 \pm 3.2$ with control IgG, $54.1 \pm 5.0$ with IgG against TRPV2) as well as skeletal muscle dystrophy, and prevented the progression of myocardial injuries in J2N-k evaluated by echocardiography and tissue Masson's trichrome staining.

These results indicate that the activation of TRPV2 contributes to abnormal $\mathrm{Ca}^{2+}$ homeostasis and muscle damage, and hint that the inhibition of this channel may be a promising therapeutic strategy for cardiomyopathy as well as muscular dystrophy. 